\title{
Anemias ferropénicas y subclínicas en indígenas muiscas de Bosa y Suba de Bogotá
}

\author{
Iron deficiency anaemias and subclinical anaemias among the muiscas \\ indigenious of Bosa and Suba in Bogota
}

Martha Castillo Bohórquez ${ }^{1}$, Ana Isabel Mora Bautista ${ }^{1}$, Ana Lucía Oliveros Rozo ${ }^{1}$.

\section{Resumen}

Objetivo. Determinar la presencia del síndrome anémico en comunidades indígenas muiscas, en hombres y mujeres entre 18 y 50 ańos ubicadas en resguardos indígenas de Bosa y Suba en la ciudad de Bogotá. Métodos. Las pruebas de laboratorio consideradas para este estudio incluyeron la valoración del hemograma automatizado, estudio de sangre periférico, ferritina sérica, Proteína C Reactiva, proteínas totales y albúminas. Este estudio es de tipo no probabilístico descriptivo; y transversal, diseńo muestral con un total de 80 muestras. Resultados. La población en estudio perteneciente a la comunidad Muisca ubicada en las localidades de Bosa y Suba de la ciudad de Bogotá, conformada por 12.5\% (10/80) hombres y $87.5 \%(70 / 80)$ mujeres, presentó niveles de ferritina bajos en un $35.7 \%(25 / 70)$ en mujeres y $40 \%(4 / 10)$ en hombres, lo que sugiere deficiencia de hierro; sin embargo, el 4.2\%(3/70) de las mujeres y el 10\% (1/10) de los hombres presentan anemia; 1 hombre y 1 mujer evidencian anemia ferropénica por tener tanto los niveles bajos de hemoglobina, hemoglobina corpuscular media, volumen corpuscular medio y ferritina; además de la presencia de deficiencia subclínica de hierro en un $34.3 \%(24 / 70)$ en mujeres y el 30\%(3/10) en hombres, con una alta probabilidad de desarrollar anemia ferropénica si no se realiza el tratamiento adecuado, estos resultados fueron reportados al personal médico de la comunidad indígena con el fin de iniciar el estudio y los tratamientos pertinentes a esta deficiencia.

Palabras Clave: Muiscas, anemia, ferropenia, inflamación, infección.

\section{Abstract}

Objective. To investigate the presence of anemic syndrome in Muisca indigenous communities, men and women between 18 and 50 years, located in indigenous territories of Bosa and Suba in Bogotá. Methods. Laboratory tests considered for this study included the assessment of automated hemogram, study of peripheral blood, serum ferritin, C-reactive protein, total protein, and albuminas. This research study is non-random, descriptive and cross-sectional, whose sample design is layered with a total of 80 samples. Results. The Muisca population under study located in the towns of Bosa and Suba in Bogotá, population consist of 12.5\% (10/80) men y 87.5\% (70/80) women presented low ferritin levels in 35.7\% (25/70) women and $40 \%$ (4/10) men , which suggests iron deficiency, however 1 man and 1 woman, show iron-deficiency anaemia, for having both levels of hemoglobin, mean corpuscular volume, mean corpuscular hemoglobin

1. Docentes investigadoras semillero ERITRON Universidad Colegio Mayor de Cundinamarca.

Correspondencia: mlcastillo@unicolmayor.edu.co

Fecha de recepción: Junio 27 de 2016 - Fecha de aprobación: Octubre 18 de 2016 
and ferritin low, this suggests the presence of subclinical iron deficiency in a $34.4 \%(22 / 70)$ women and 30\%(3/10) men, therefore the future possibility of iron-deficiency anaemia but the causes of iron deficiency are corrected.

Keywords: Muiscas, Anaemia, Ferropenia, Inflammation, Infectión

\section{Introducción}

Colombia es un país que se caracteriza por su riqueza multicultural y diversidad étnica. Las comunidades indígenas, por su vulnerabilidad frente a las diversas problemáticas del país, la violencia, la pobreza, la falta de oportunidades en su lugar de origen, han migrado a las grandes ciudades, generalmente llegando a engrosar los cinturones de pobreza, y como consecuencia, surgen problemas adicionales, tales como la desnutrición y dificultad en el acceso adecuado a los servicios de salud.(1)

El grupo de investigación ERITRON, preocupado por esta situación, realiza un estudio que determina la presencia del síndrome anémico en comunidades indígenas muiscas, en hombres y mujeres entre 18 y 50 años ubicadas en resguardos indígenas de Bosa y Suba en la ciudad de Bogotá.

Según la organización nacional de indígenas (ONIC), en Colombia se encuentran cerca de 102 comunidades, la mayoría de la población se haya ubicada en los departamentos de la Guajira, Cauca, Nariño y Córdoba. En Bogotá se organizan en cabildos, como Muisca de Bosa, Muisca de Suba, Mayor Inga Kichwa de Bogotá "Camainkibo", Indígena Ambika Etnia Pijao, Indígena de Bogotá, entre otros (1).

La población indígena se ve afectada en su estado de salud debido a varios factores como la situación socioeconómica, nutrición inadecuada, la presencia de contaminación de los recursos y la violencia; factores que llevan a la migración a las grandes ciudades, en condiciones poco favorables, con escasa posibilidad de mejorar su condición de vida. Dicha población, además, utiliza medicina tradicional con chamanes para atender los problemas de salud. (2).

En Colombia se han establecido marcos legales y constitucionales que promueven la justicia social y el derecho de las comunidades indígenas, reglamentadas por el convenio 169 de la Organización Internacional del Trabajo (OIT), la Declaración de las Naciones Unidas sobre los Derechos de los pueblos indígenas, la Constitución de 1991, el Decreto de 1396 de 1996 y el Decreto 543 del 2011 donde el Estado reconoce y protege la diversidad étnica y cultural, también constituye que la salud sea un derecho esencial sin discriminación y se brinde como un servicio público para mejorar la calidad de vida de los ciudadanos (3) (4) (5) (6) (7) (8).

Cuando los requerimientos fisiológicos del hierro no son los necesarios o hay un gasto excesivo, conlleva a anemias o deficiencias subclínicas de hierro, que pueden también ser provocadas por enfermedades crónicas tales como infecciones, inflamaciones y neoplasias que no permiten una adecuada absorción del hierro (9).

Teniendo en cuenta que la deficiencia de hierro es un problema de salud pública mundial (10) son muy escasos los estudios realizados en Colombia para conocer los estados de salud de los integrantes de las comunidades indígenas, relacionados principalmente a deficiencias subclínicas y anemia ferropénica.

Por lo anterior es importante conocer cuál es el estado de salud de las comunidades indígenas muiscas en la periferia de Bogotá, mediante pruebas de laboratorio que evalúan la presencia 
de anemias ferropénicas de los participantes y de esta manera generar intervención por parte de entidades gubernamentales propias de estos grupos y del Estado, que contribuyan al mejoramiento de calidad de vida de esta población y que se puedan generar programas de atención primaria para disminuir las deficiencias de este biometal y la tasa de morbimortalidad de estas comunidades indígenas.

A nivel mundial, las comunidades indígenas son vulnerables y olvidadas, como se evidencia en los diferentes estudios encontrados. Por ejemplo, en el artículo titulado "Pueblos indigenas y sistemas de información en salud: La variable étnica en seis provincias argentinas", en el cual se analizaron diferentes comunidades indígenas, se ponen en evidencia las altas tasas de mortalidad materna, desnutrición y enfermedades infecciosas, y se sugiere una implementación de programas piloto en epidemiología y salud indígena (11).

En el estudio titulado "Perfil de hierro en menores de seis comunidades indígenas residentes en la ciudad de Cali, Colombia" se evidenció una prevalencia de anemia con alteración en las reservas de hierro del 25,8\%, siendo los Inga y los Yanacona las comunidades indígenas más afectadas (12).

El estudio realizado por Rosique y colaboradores, menciona que las comunidades indígenas se han visto afectadas gradualmente por aspectos sociales, políticos, económicos, entre otros, como lo son el conflicto armado colombiano que los lleva en muchas ocasiones a desplazarse de su hábitat natural, y por tanto, a perder todos los beneficios que tenían en sus tierras, lo que a su vez lleva a que no cuenten con los medios suficientes para subsistir, con la consiguiente falta de recursos y posterior déficit alimentario, económico y social.

En el trabajo con los indígenas Embera, procedentes de frontino Antioquia, encontraron que todos estos factores anteriormente mencionados alteran el correcto funcionamiento y supervivencia de estas comunidades, pues ya no se dedican a sus labores cotidianas como la pesca, caza o recolección, sino que tienen que recurrir a otro tipo de actividades como la elaboración de artesanías que venden con mucha dificultad, para poder llevar un sustento a sus hogares. Esta situación, junto con la escasez de recursos, conlleva a que estas personas no se alimenten adecuadamente lo que se evidencia en el aumento gradual en la desnutrición de esta etnia colombiana, afectando principalmente a los niños quienes son los que deben consumir alimentos apropiados que les aporten vitaminas y una dieta balanceada para el correcto crecimiento (13).

En comunidades indígenas no intervenidas, con sujetos no inmunizados o con inmunidad debilitada, casos tales como infantes y adultos mayores se convierten en focos potencialmente infectocontagiosos y de vulnerabilidad aumentada, transformándose en reservorios de infección, con elevadas tasas de mortalidad. En el contexto colombiano, la población que habita los departamentos de Vaupés, Vichada, Guajira, Guainía y Amazonas con marcada concentración se constituyen en las de mayor vulnerabilidad (14).

El presente trabajo no es ajeno a la problemática mencionada y pretende continuar con estos tipos de investigación, cuyo objetivo es detectar la presencia de anemias ferropénicas y deficiencias subclínicas de hierro en comunidades indígenas Muiscas de las localidades de Bosa y Suba de la ciudad de Bogotá.

\section{Materiales y métodos}

La muestra del estudio correspondió a hombres y mujeres que cumplieran los siguientes criterios de inclusión: ser mayor de 18 años, pertenecer a las comunidades indígenas Muiscas de Suba y Bosa, haber autorizado su participación en el estudio firmando el consentimiento informado. Se analizaron 80 muestras de sangre total y suero de hombres y mujeres adultos indígenas Muiscas colombianos. La información se recolectó a partir de los resultados de las pruebas de laboratorio: 
Tabla 1. Distribución por género

\begin{tabular}{ccccc}
\hline Total de muestra & mujeres & $\%$ mujeres & hombres & $\%$ hombre \\
\hline 80 & 70 & 87.5 & 10 & 12.5 \\
\hline
\end{tabular}

Tabla 2. Porcentaje de anemias ferropénicas/deficiencia subclínica de hierro en mujeres

\begin{tabular}{cccccc} 
ferritina baja & $\begin{array}{c}\text { \% ferritina } \\
\text { baja }\end{array}$ & anemia & $\begin{array}{c}\text { anemia deficiencia } \\
\text { de hierro }\end{array}$ & $\begin{array}{c}\text { \% anemia deficiencia } \\
\text { de hierro }\end{array}$ & $\begin{array}{c}\text { \% Deficiencia } \\
\text { subclínica de hierro }\end{array}$ \\
\hline 25 & 35.7 & 3 & 1 & 1.4 & 34.3 \\
\hline
\end{tabular}

Tabla 3. Porcentaje de anemias ferropénicas/deficiencia subclínica de hierro en hombres

\begin{tabular}{cccccc} 
ferritina baja & $\begin{array}{c}\text { \% ferritina } \\
\text { baja }\end{array}$ & anemia & $\begin{array}{c}\text { anemia deficiencia } \\
\text { de hierro }\end{array}$ & $\begin{array}{c}\% \text { anemia deficiencia } \\
\text { de hierro }\end{array}$ & $\begin{array}{c}\text { \% Deficiencia } \\
\text { subclínica de hierro }\end{array}$ \\
\hline 4 & 40 & 1 & 1 & 10 & 30 \\
\hline
\end{tabular}

Tabla 4. Porcentaje de PCR aumentada

\begin{tabular}{ccccc}
\hline Total de muestra & mujeres & $\%$ mujeres & hombres & $\%$ hombres \\
\hline 80 & 6 & 8.6 & 2 & 20 \\
\hline
\end{tabular}

Hemograma de tercera generación: muestras de sangre anticoagulada recolectada por flebotomía en tubo tapa lila con EDTA, las cuales se procesaron en el equipo de hematología automatizado Mildray BC3000 plus.

Estudio de sangre periférica: los extendidos de sangre valorando la morfología por microscopía óptica según protocolo estandarizado.

Ferritina sérica: se evaluó la presencia y cantidad de dicha proteína mediante la técnica "Human Ferritin Enzyme Immunoassay Test Kit", que sugiere valores de referencia para mujeres de $20 \mu \mathrm{g} / \mathrm{L}$ a $200 \mu \mathrm{g} / \mathrm{L}$ y hombres de $20 \mu \mathrm{g} / \mathrm{L}$ a $250 \mu \mathrm{g} / \mathrm{L}$.

PCR (Proteína C Reactiva): mediante el test rápido para la determinación cualitativa y semicuantitativa de Proteína $\mathrm{C}$ por aglutinación de las partículas de látex en portaobjetos, que sugiere valores normales hasta $6.0 \mathrm{mg} / \mathrm{l}$. Es muy importante destacar que después del análisis de estas muestras, se realiza todo el proceso de inactivación y eliminación del material de riesgo biológico en el lugar de procesamiento.

Proteinas séricas: se valoró la presencia de estas proteínas por método colorimétrico.
Para el análisis estadístico se utilizó Microsoft Excel versión 2007. Las variables cuantitativas se analizaron con medidas de tendencia central como el promedio y se describieron por medio de frecuencias absolutas y porcentuales. Se realizó un análisis de tipo descriptivo, correlacional, análisis de varianza y pruebas no paramétricas utilizando pruebas estadísticas como chi-cuadrado, prueba de Fischer y la correlación de Spearman. Un P valor menor de 0.05 se consideró significativo.

\section{Resultados}

En el estudio participaron dos comunidades muiscas de Suba y Bosa, a continuación, se pueden observar los resultados de las pruebas

\section{Discusión}

Los registros que se encuentran acerca de la incidencia de anemias en comunidades indígenas en Colombia son pocos. Este trabajo contribuye en la construcción de estadísticas en salud en la ciudad de Bogotá, permitiendo la implementación de programas de salud diseñados específicamente para la población de estudio. 
En la publicación denominada "Pueblos indígenas y sistemas de información en salud: la variable étnica en seis poblaciones argentinas" de 2011, se describe que las comunidades indígenas no cuentan con registros que se puedan analizar y permitan diseñar políticas en salud, por lo que se sugiere la implementación de programas piloto en epidemiología y salud indígena (10). En la presente investigación se evidenció la escasa información acerca de las condiciones de salud de estas comunidades en Colombia.

En el trabajo de campo, el grupo de investigación observó que las personas pertenecientes a las comunidades Muiscas de las localidades de Bosa y Suba, viven en condiciones de pobreza, aspecto que conlleva a que el nivel nutricional no sea el adecuado y la atención en salud sea deficiente, datos que se relacionan con el estudio de Rosique y colaboradores que describe el estado nutricional y hábitos alimentarios en Indígenas Embera de Colombia (13).

Esimportante informar a los entes de salud y nutrición correspondientes acerca de establecer la identificación temprana de anemias ferropénicas, teniendo en cuenta los índices de desplazamiento actual y los resultados evidenciados en esta investigación donde se encontró deficiencia subclínica de hierro en mujeres de $34.3 \%$ y en hombres $30 \%$ que deben recibir tratamiento antes que desarrollen anemia ferropénica, y anemia ferropénica en mujeres $1.4 \%$ y en hombres $10 \%$,la población masculina no es representativa y se debe realizar investigaciones con muestreo mayor para corroborar este dato.

Otro aspecto importante que se debe tener en cuenta es la presencia de procesos inflamatorios o infecciosos en la comunidad en estudio, teniendo en cuenta que el $8.6 \%$ de mujeres y $20 \%$ en hombres cursa con PCR elevada, que se correlaciona con los hallazgos de la investigación en indígenas de las comunidades de los departamentos de Amazonas, Caquetá y Cundinamarca $(15,16)$.

En efecto, se describe un estudio en comunidades indígenas en los departamentos de Amazonas,
Caquetá y Cundinamarca, se encontró anemia en los participantes de los departamentos de Amazonas y de Caquetá, más no se describe en Cundinamarca. Este dato se correlaciona con la baja incidencia de anemia en las comunidades muiscas de Bosa y Suba. $(15,16)$. Sin embargo, la incidencia de ferritina baja, sí aumenta en los dos estudios, posiblemente por cambios en hábitos alimenticios que han adquirido por la migración a las ciudades, datos que hay que tener presente para prevenir estados subclínicos de anemias ferropénicas.

El valor de ferritina baja con hemoglobinas normales indica deficiencias subclínicas de hierro lo cual requiere de la intervención médica inmediata para prevenir la anemia. Por otra parte, al ser la ferritina una proteína de fase aguda y analizando el porcentaje de personas con PCR elevada, podría enmascararse algunos estadios subclínicos que no se evidenciaron en la investigación $(15,16)$.

\section{Conclusiones}

La población en estudio perteneciente a la comunidad Muisca ubicada en las localidades de Bosa y Suba de la ciudad de Bogotá, presentó niveles de ferritina bajos en mujeres $35.7 \%$ $(25 / 70)$ y hombres $40 \%(4 / 10)$, lo que sugiere deficiencia subclínica de hierro, en corto tiempo puede desarrollar anemia ferropénica con sus consecuencias. El 1.4 \% (1/70) en mujeres y 10\% $(1 / 10)$ en hombres presenta anemia ferropénica.

Los resultados se entregaron a los líderes de las comunidades, quienes consultaron con los entes de salud a los cuales pertenecen, para conseguir un adecuado manejo de las personas que presentaron algún resultado alterado y de esa manera contribuir al beneficio de los participantes en atención primaria en salud.

La población en estudio pertenece a comunidades de bajos recursos, por ello es importante hacer campañas de educación que fomenten hábitos 
alimenticios apropiados para evitar deficiencias subclínicas de hierro ya que pueden llegar a desarrollar anemias ferropénicas.

El grupo Eritron está comprometido con la consecución de nuevos proyectos en población vulnerable, teniendo en cuenta los resultados hallados en este estudio como respuesta a las solicitudes de las comunidades y como aporte en estudios epidemiológicos y de salud pública.

Se sugieren desarrollar programas de salud pública, reconociendo las particularidades de cada grupo étnico y así diseńar e implementar procesos concertados que conlleven a la prevención y control de las enfermedades de interés para la comunidad.

\section{Referencias}

1. El Trabajo del ACNUR con Pueblos Indígenas. [place unknown: publisher unknown]. Documentos - Pueblos Indígenas; 2013 Apr 18 [cited 2016 May 26]. Available from: http://www.acnur.org/t3/pueblos-indigenas/documentos/.

2. Poveda E., Cuartas A., Guarín S., Forero Y., Villarreal E. Estado de los micronutrientes hierro y vitamina $\mathrm{A}$, factores de riesgo para las deficiencias y valoración antropométrica en niños preescolares del municipio de Funza, Colombia. Biomédica [Internet]. 2007 Mar [cited 2016 May 26] ; 27( 1 ): 76-93. Available from: http:// www.scielo.org.co/scielo.php?script=sci_arttext\&pid=S0120 41572007000100008\&lng=en.

3. Pueblos indígenas dialogo entre culturas. Bogota Colombia: PNUD; 2012 Apr 12. [cited 2016 May 26]. Available from: http://www.aecid.org.co/recursos_user/publicaciones\%20 aecid/cuadernoindigenas.pdf.

4. User S. Secretaria Distrital de Integracion Social. [place unknown: publisher unknown]. Políticas públicas para los pueblos Indígenas, para la población Afrodescendientes; y lineamientos de políticas para las comunidades Raizales, Rrom o Gitano en el Distrito capital; 2015 [cited 2016 May 26]. Available from: http://www.integracionsocial.gov.co/index. php/politicas-publicas/indigenas-y-afrodescendientes.

5. Declaración de las Naciones Unidas sobre los derechos de los pueblos indígenas. [place unknown]: Publicado por las Naciones Unidas 07-58684-marzo de 2008—3, 000; 2008. [cited 2016 May 26]. Available from: http://www.un.org/esa/ socdev/unpfii/documents/DRIPS_es.pdf.

6. DECRETO 1396 DE 1996. (agosto 8) Diario Oficial No. 42.853, de 12 de agosto de 1996 MINISTERIO DEL INTERIOR, (1996 Aug 8).
7. OPIAC. OPIAC. [place unknown: publisher unknown]. Comisión de Derechos Humanos Pueblos Indígenas, condena masacre contra comunidad Nasa; 2015 Apr 20 [cited 2016 May 26]. Available from: http://www.opiac.org.co/index.php/ noticias/nacionales/333-comision-de-derechos-humanospueblos-indigenas-condena-masacre-contra-comunidad-nasa.

8. OIT. Convenio 16907 sobre pueblos indigenas y triba en paises independientesl. Segunda edición 2007 ed. [place unknown]: OIT; 2007. [cited 2016 May 26]. Available from: http://www. oit.org.pe/WDMS/bib/publ/libros/convenio_169_07.pdf.

9. Rodak,B. Hematología fundamentos y aplicación clínica. Editorial medica Panamericana ed. Mexico: [publisher unknown]; 2014. [cited 2016 May 23]. Available from: http://RodakBernadette . Hematología fundamentos y aplicación clínica, cuarta edición, editorial medica Panamericana, 2014. Capitulo 19 pag 289-306, ISBN 978-607-9356-16-3, www.isbn-internacional.org.

10. Coy L., Castillo M., Mora A., Oliveros A., Vélez Z. Estrategias diagnosticas utilizadas para detectar deficiencias de hierro subclínicos y asociadas a enfermedades crónicas. Nova; 2005; 3(4):1-116.

11. Cuyul A, Rovetto M, Specogna M. Pueblos indígenas y sistemas de información en salud: La variable étnica en seis provincias argentinas. Revista Argentina de Salud Pública [En línea], 2011 [Revisado 14 de Septiembre de 2011]; 2(7): 12-18. Disponible en: http://www.msal.gov.ar/rasp/rasp/ articulos/volumen7/art-originales pueblosindigenas.pdf

12. Bolaños M, Echeverry O, Bermúdez A, et al. Perfil del hierro en menores de seis comunidades indígenas residentes en la ciudad de Cali, Colombia. Universidad del Valle, Facultad de Salud, Escuela de Bacteriología y Laboratorio Clínico. Grupo de investigación: "INBIOMIC" y "CALIMET", 2011; [Revisado 27 de Enero de 2012]. Disponible en: http://salud.univalle.edu. co/simposio/index.php/simposio/13simposio/paper/view/59/0

13. Rosique J, Restrepo M, Manjarrés M, Gálvez A, Santa M. Estado nutricional y hábitos alimentarios en Indígenas Embera de Colombia. Rev. chilena de nutrición [En línea], 2010 [Revisado 2 de Septiembre de 2011]; 37 (3): 270 - 280. Disponible en: http://www.scielo.cl/pdf/rchnut/v37n3/art02.pdf

14. Departamento Nacional de Planeación. Compendio de Políticas Culturales, Ministerio de Cultura, República de Colombia, Bogotá. [En línea], 2010, págs. 371-383. [Revisado 11 de abril de 2012]. Disponible en: http://www.dnp.gov.co/ LinkClick.aspx?fileticket=CpCS1dVTQf4\%3D\&tabid=273

15. Castillo M, Oliveros A, Mora A, Description of the Health Status of Colombian Indigenous Communities Through Basic Laboratory Test. Journal of Life Sciences, 2014; Vol 8 (9) 789793 www.unicolmayor.edu.co ISSN 1934-7391, USA Faculty of Health Sciences, Universidad Colegio Mayor de Cundinamarca, Calle 28 No. 5B-02, Bogotá, D.C. 110911, Colombia

16. Campuzano S, Mejía Flórez D, Madero Ibarra C, Pabón Sánchez P. Determinación de la calidad microbiológica y sanitaria de alimentos preparados vendidos en la vía pública de la ciudad de Bogotá DC. Nova. 2015;13(23):81-92. 